\title{
Propiedades psicométricas de la Escala de Desmoralización (DS-II versión español-Colombia] para pacientes oncológicos que reciben cuidado paliativo
}

Psychometric Properties of Demoralization Scale [DS- || Spanish Uersion-Colombia) for Oncologic Patients in Palliative Care

\section{Propriedades Psicométricas da Escala de Desmoralização (DS-II versão espanhol- Colômbia] para pacientes oncológicos que recebem cuidado paliativo}

\author{
Ximena Palacios-Espinosa \\ Universidad del Rosario \\ Ricardo Sánchez Pedraza \\ Instituto de Investigaciones Clínicas, Facultad de Medicina, Universidad Nacional de Colombia. \\ Instituto Nacional de Cancerología \\ Carlos Rodríguez \\ Instituto Nacional de Cancerología
}

Doi: https://doi.org/10.12804/revistas.urosario.edu.co/apl/a.8408

\section{Resumen}

La desmoralización es un síndrome psiquiátrico frecuente en pacientes oncológicos en cuidados paliativos, por lo cual su medición es pertinente y relevante en el ámbito de la psicooncología. El objetivo de este estudio fue analizar las propiedades psicométricas de la Escala de Desmoralización II (DS-II) en Colombia, en 516 pacientes adultos con cáncer en estadios avanzados. Se realizó adaptación transcultural de la DS-II y se determinaron sus propiedades psicométricas desde las teorías clásicas de medición y la teoría de respuesta al ítem (análisis de Rasch). Se analizaron validez de constructo, consistencia interna, validez de criterio y confiabilidad. El análisis factorial exploratorio mostró tres dominios respaldados por análisis factorial confirmatorio que además mostró un ajuste aceptable de la DS-II. Los resultados indicaron que la versión español-Colombia de la DS-II es confiable (alfa de Cronbach $=0.87 \mathrm{y}$ lambda de Gutman $=0.88$ ) y útil para identificar niveles intermedios de desmoralización sin uno de los ítems.

Ximena Palacios-Espinosa ORCID ID: https://orcid.org/0000-0002-9503-139X

Ricardo Sánchez Pedraza ORCID ID: https://orcid.org/0000-0002-7540-4734

Carlos Rodríguez ORCID ID: https://orcid.org/0000-0002-7871-0155

Nota: La filiación completa de Ricardo Sánchez Pedraza es Instituto de Investigaciones Clínicas, Facultad de Medicina, Universidad Nacional de Colombia. Instituto Nacional de Cancerología

Los profesores agradecen a Ana María Gómez y Juan Sebastián Botero; a la psicóloga Jenny Fernanda Vargas y a los estudiantes del Programa de Psicología de la Universidad del Rosario, por su contribución en alguna(s) de las fases del desarrollo del proyecto: Tatiana Castillo; Lady Mayori Guzmán; María Camila Solano; Katheryn Hernández; Luisa María Forero Vela; Juliana Ocampo Palacio; Daniela Carolina Obando González; Angie Marcela Benavides Contento y Mariana Parra Cruz.

Dirigir correspondencia a Ximena Palacios-Espinosa. Correo electrónico: ximena.palacios@urosario.du.co

Para citar este artículo: Palacios-Espinosa, X., Sánchez Pedraza, R., \& Rodríguez, C. (2020). Propiedades psicométricas de la Escala de Desmoralización (DS-II versión español-Colombia) para pacientes oncológicos que reciben cuidado paliativo. Avances en Psicología Latinoamericana, 38(3), 1-18. https://doi.org/10.12804/revistas.urosario.edu.co/apl/a.8408 
Además, se discuten otros resultados relacionados con la confiabilidad test-retest y la sensibilidad, así como su uso potencial en la población estudiada.

Palabras clave: desmoralización; estudios de validación; reproducibilidad de los datos; cuidados paliativos; cáncer.

\section{fibstract}

Demoralization is a frequent psychiatric syndrome in cancer patients in palliative care, so its measurement is relevant in the field of Psycho-oncology. The objective of this study was to analyze the psychometric properties of the Demoralization-Scale II (DS-II) applied in Colombia to 516 adult patients with cancer in advanced stages. Transcultural adaptation of the DS-II was performed, and its psychometric properties were determined from the classical theories of measurement and Item Response Theory (Rasch analysis). Content validity, internal consistency, criterion validity, and reliability were analyzed. The exploratory factor analysis showed three domains backed by confirmatory factor analysis that also showed an acceptable adjustment of the DS-II. The results indicated that the DS-II (Spanish-Colombia version) is reliable (Cronbach's alpha $=0.87$ and lambda de Gutman $=0.88$ ) and useful for identifying intermediate levels of demoralization without one of the items. Other results related are also discussed (test-retest reliability and sensitivity, and its potential use in the studied population).

Keywords: Demoralization; validation studies; reproducibility of results; palliative care; neoplasms.

\section{Resumo}

A desmoralização é uma síndrome psiquiátrica frequente em pacientes oncológicos em cuidados paliativos, pelo qual sua medição é pertinente e relevante no âmbito da psico-oncologia. O objetivo deste estudo foi analisar as propriedades psicométricas da Escala de Desmoralização II (DS-II) ao ser aplicada na Colômbia, em 516 pacientes adultos com cancro em estádios avançados. Se realizou adaptação transcultural da Escala e se determinaram suas propriedades psicométricas desde a teoria clássica de medição e a teoria de resposta ao item (análise de Rasch). Se analisaram a validade de constructo, consistência interna, validade de critério, confiabilidade. A análise fatorial exploratória mostrou um ajuste aceitável da Escala. Os resultados indicaram que a DS-II (versão espanhol-Colômbia) é confiável (alfa de Cronbach $=0.87$ e Lambda de Gutman $=0.88$ ) e útil para identificar níveis intermédios de desmoralização, sem um dos itens. Se discutem outros resultados relacionados com a confiabilidade teste re teste e a sensibilidade, assim como seu uso potencial na população estudada.

Palavras-chave: desmoralização; estudos de validação; reprodutibilidade dos dados; cuidados paliativos; cancro.

La desmoralización es un síndrome psiquiátrico, descrito por David Kissane, frecuente en pacientes oncológicos que reciben cuidado paliativo, que cobra cada vez mayor importancia entre los fenómenos psicológicos que se presentan en esta población (Grassi \& de Figueiredo, 2018; Robinson et al., 2015). Sus orígenes como concepto están en la psicoterapia existencial (Robinson et al., 2016b). Actualmente, la desmoralización se refiere a "un estado psiquiátrico en el que la desesperanza, la impotencia, la pérdida del sentido y el distrés existencial son el núcleo del fenómeno" (Kissane et al., 2001, p. 13), que debe persistir más de dos semanas y que puede ser diagnosticado con base en 6 criterios: (1) Síntomas afectivos o distrés existencial, incluyendo desesperanza o pérdida de sentido y propósito de la vida - La desesperanza es el elemento fundamental de la desmoralización (Clarke \& Kissane, 2002) - ; (2) Actitudes cognoscitivas de pesimismo, impotencia, sentido de estar atrapado, fracaso personal o ausencia de futuro o futuro sin sentido; (3) Ausencia conativa para dirigir o afrontar de manera diferente; (4) Características asociadas con la alienación social, el aislamiento o la ausencia de apoyo; (5) Fluctuación en la intensidad de las emociones, y (6) Ausencia de trastorno depresivo mayor primario (Kissane et al., 2001). Este síndrome es el resultado de la confrontación con eventos estresantes que interfieren en los 
sentimientos de control y competencia durante todo el proceso de la enfermedad (Grassi \& de Figueiredo, 2018). Dada la relevancia clínica de la desmoralización y el interés por su identificación en el marco de los cuidados paliativos oncológicos, es necesario evaluarla de manera precisa para planear una intervención igualmente precisa. Kissane et al. (2004) diseñaron una medida específica para pacientes con cáncer avanzado: la Escala de Desmoralización (DS), cuyo uso se ha extendido ampliamente y ha sido traducida en diversos idiomas, entre ellos el mandarín (Hung et al., 2010), el alemán (Mehnert et al., 2011), el italiano (Costantini et al., 2013) y el español de España (Rudilla et al., 2016). Su versión en inglés ha sido validada para diversos contextos culturales, por ejemplo en Irlanda (Mullane et al., 2009).

La DS original está constituida por 22 ítems y tiene 5 subescalas: (1) Pérdida de significado y propósito; (2) Desamparo; (3) Disforia; (4) Descorazonamiento, y (5) Sentido de fracaso (Robinson et al., 2016b). Recientemente, estos autores publicaron una versión refinada y revalidada de esta escala: la DS-II. El proceso de validación se realizó con 211 pacientes, la mayoría de ellos (89.6\%) con cáncer y otros con enfermedades progresivas avanzadas diferentes al cáncer como falla renal $(0.5 \%)$, enfermedades neurológicas (4.2\%) y enfermedades cardiorespiratorias $(5.7 \%)$. En el análisis factorial exploratorio, el instrumento mostró una estructura con dos factores: (1) significado y propósito y (2) distrés y habilidad de afrontamiento. Las dos subescalas, cada una de 11 ítems, mostraron buen ajuste y unidimensionalidad. Sin embargo, la decisión basada en el análisis de Rash fue proponer una versión de 16 ítems (DS-II), con una distribución de 8 en cada subescala, que mostró buen ajuste y unidimensionalidad, así como consistencia interna tanto en la escala total -Índice de Separación de Personas (PSI) $=0.79 ; \alpha=0.89-$, como en cada una de las subescalas: significado y propósito ( $\mathrm{PSI}=$ $0.72 ; \alpha=0.89)$ y distrés y habilidad de afrontamiento $(\mathrm{PSI}=0.73 ; \alpha=0.87)$, y validez convergente con otros instrumentos psicométricos que evalúan carga, calidad de vida, bienestar existencial, depresión y actitudes hacia el final de la vida (Robinson et al., 2016a). Adicionalmente la escala de respuesta fue ajustada a 3 puntos para facilitar su uso clínico, debido al deterioro propio de los pacientes con estados avanzados de enfermedad. Se espera que su aplicación permita el diagnóstico oportuno de la desmoralización para diseñar y desarrollar una intervención efectiva (Robinson et al., 2016b).

Hasta donde tenemos conocimiento, no existe una validación de la escala DS-II en idioma español. En consecuencia, el objetivo del presente estudio fue analizar las propiedades psicométricas de este instrumento en Colombia, en una subpoblación específica: pacientes adultos con cáncer en estadios avanzados.

\section{Método}

\section{Participantes}

Los pacientes del estudio fueron tomados del servicio de cuidados paliativos del Instituto Nacional de Cancerología de Colombia, durante el periodo de agosto de 2017 a octubre de 2018. Se tuvieron en cuenta los siguientes criterios de inclusión: tener diagnóstico confirmado de cáncer, presentar estadificación de tres o más, ser mayor de edad, tener nacionalidad colombiana y ser hispanoparlante. No desear participar en el estudio o la dificultad para diligenciar el instrumento por compromiso del estado general de salud, se tomaron como criterios de exclusión. Estos criterios se aplicaron en un muestreo no probabilístico secuencial por conveniencia, a los primeros 545 pacientes detectados en el periodo en el que se realizó el estudio. Un total de 23 pacientes se excluyeron por presentar compromiso del estado general que impidió el trámite del instrumento; seis pacientes fueron excluidos por no aceptar participar en el estudio. 


\section{Instrumentos}

Escala de Desmoralización II (DS-II) (Robinson et al., 2016a; 2016b): constituida por 16 ítems, en escala Likert de 3 categorías, dimensiona la frecuencia de cada uno de estos en las últimas dos semanas, y consta de dos subescalas: (1) significado y propósito y (2) distrés y habilidad de afrontamiento. El puntaje va de 0 a 32 con los siguientes puntos de corte: $0-3$ (bajo), 4-10 (moderado) $\mathrm{y} \geq 11$ (alto) (Robinson et al., 2016a).

Inventario de Depresión de Beck - segunda edición (BDI-II) (Beck et al., 1996): versión en español de Sanz et al. (2003), actualmente representada por el Departamento I+D Pearson Clinical \& Talent Assessment. Está constituido por 21 grupos de afirmaciones. La instrucción es señalar la afirmación de cada grupo que describe mejor la manera en que la persona se ha sentido durante las últimas dos semanas. Este instrumento evalúa la gravedad de la sintomatología depresiva en adultos y adolescentes con una edad mínima de 13 años y específicamente dentro del presente estudio, se utilizó para analizar la validez de criterio concurrente, previa consulta con el autor del instrumento y basado en el uso del mismo en otros estudios (Costantini et al., 2013; Kissane et al., 2004; Mullane et al., 2009). La puntuación va de 0-63 y los puntos de corte que indican la presencia y gravedad de la depresión: 0-13 (mínima), 14-19 (leve), 20-28 (moderada) y 29-63 (grave). El BDI-II presenta una confiabilidad de $\alpha=0.87$.

\section{Procedimiento}

El estudio fue evaluado y aprobado por el Comité de Ética del Instituto Nacional de Cancerología de Colombia. Todos los participantes dieron su consentimiento informado verbal para participar.

El estudio se desarrolló en varias fases adaptadas de la propuesta de Carvajal et al. (2011). En consecuencia, la primera fase comprendió el proceso de traducción y adaptación transcultural, con autorización del doctor David Kissane y la participación de profesionales de la salud bilingües, a partir de la versión original de la DS-II. Se realizaron dos traducciones conceptuales de la versión original de la escala al español de Colombia. Una vez realizadas las traducciones, se conformó un comité de expertos en el cual se evaluó la equivalencia semántica de las dos versiones. Adicionalmente, las dudas sobre el sentido de algunos ítems fueron directamente aclaradas con el autor. Con base en los resultados de la revisión semántica, un comité revisor diseñó la primera versión definitiva del instrumento, que fue aplicada en el estudio piloto.

En la segunda fase se aplicó la prueba piloto a 20 pacientes, tanto hospitalizados como ambulatorios. El comité revisor efectuó el análisis de los resultados obtenidos en este estudio y tras la realización de los ajustes pertinentes, diseñó la versión definitiva de la escala en español de Colombia, que fue comentada con el profesor Kissane (ver Anexo).

En la tercera fase, se procedió a aplicar la DS-II (versión en español-Colombia) en forma de entrevista personal. A un grupo de 95 pacientes se les aplicó el BDI-II junto con la DS-II (versión en español-Colombia) con el propósito de evaluar la validez de criterio concurrente de la DS-II (versión en español - Colombia); a otro grupo de 100 pacientes se les aplicó nuevamente la DS-II entre 3 y 7 días después de la primera aplicación con el fin de determinar la confiabilidad del instrumento.

\section{Análisis estadístico}

Para el componente descriptivo se usaron porcentajes para variables categóricas, y medias o medianas para variables continuas, junto con sus correspondientes medidas de variabilidad: desviación estándar (DE) o rango inter-cuartílico (RIC). Para la determinación de las propiedades psicométricas del instrumento se hizo un abordaje desde la teoría clásica de medición y desde la teoría de respuesta al ítem (análisis de Rasch). Para establecer la 
validez de constructo se utilizaron métodos de análisis factorial (factores principales), recurriendo a una matriz de correlación policórica (considerando la naturaleza ordinal de los ítems). Los diagnósticos prefactorización de la matriz se efectuaron con la prueba de esfericidad de Bartlett y el test de Kaiser-Meyer-Olkin (KMO). La determinación del número de factores se efectuó con el análisis del gráfico de sedimentación de valores propios y test de análisis paralelo. Para el análisis factorial confirmatorio se calcularon medidas de bondad de ajuste con la biblioteca Lavaan del sistema R, utilizando una muestra aleatoria de 320 pacientes.

La consistencia interna del instrumento se evaluó calculando para la escala total y para cada dominio o factor, los coeficientes alfa de Cronbach y lambda de Guttman.

La validez de criterio concurrente se evaluó con coeficientes de correlación de Pearson entre los puntajes totales de la DS-II (versión español-Colombia) y el BDI-II, y entre los puntajes de los factores de la DS-II (versión español-Colombia) y el puntaje total del BDI-II. Para evaluar la confiabilidad mediante el uso de medidas repetidas se calculó el coeficiente de correlación-concordancia de Lin (Lawrence \& Lin, 1989).

Finalmente, se hizo análisis de Rasch en el que se verificó la unidimensionalidad de la DS-II (versión español-Colombia), a través de un análisis de componentes principales de los residuos. Con este procedimiento se esperan porcentajes de varianza cruda explicada por la medición $>40 \%$ y que el eigenvalue de varianza no explicada correspondiente al primer contraste sea $<2$ (Linacre, 2006). Otro indicador de unidimensionalidad son valores de cuadrado medio de infit entre 0.6 y 1.5 y desviación estándar de coeficientes estandarizados $<2$.

Se estimaron medidas de separación, buscando una aproximación a la confiabilidad de personas e ítems. Esta estimación representa la cantidad de niveles en los que pueden agruparse ítems e individuos. Se consideran satisfactorios niveles de confiabilidad las medidas de separación
$>2$ para personas y $>3$ para ítems (Dimitirov \& Smith, 2006).

Se examinó la calidad del ajuste de cada uno de los ítems del instrumento al modelo. Para esto se realizó la estimación del cuadrado medio (MSQ) (Wackerly et al., 2013), de los parámetros estadísticos de ajuste outfit (ajuste sensible a los casos atípicos: outliers) y el ajuste sensible a los patrones internos (infit) (Fisher \& Molenaar, 1995; Fox \& Bond, 2015). Con respecto a la calidad de la medición de categorías de respuesta de los ítems, se evaluó el orden de estas y se realizaron sus curvas de probabilidades (Wackerly et al., 2013). Adicionalmente, se utilizó como herramienta de análisis gráfico el mapa de Wright.

\section{Resultados}

\section{Adaptación transcultural}

El proceso de adaptación transcultural se realizó con 20 pacientes con diagnóstico de cáncer en estadio avanzado ( 15 ambulatorios, 5 hospitalizados), utilizando los mismos criterios de inclusión y exclusión de los 516 pacientes de la muestra definitiva. La media de edad fue de 54.7 años; 12 $(60 \%)$ mujeres y $8(40 \%)$ hombres; la mayoría pertenecientes a estrato socioeconómico bajo (ESE) -1 y 2-; procedentes de zona urbana, de diferentes regiones del país y niveles educativos bajos.

Se diseñó un instrumento basado en los lineamientos de la EORTC (Dewolf et al., 2009), constituido por dos partes. La primera buscaba registrar información general del participante (edad, sexo, profesión o empleo, escolaridad, ESE, procedencia (rural o urbana), diagnóstico, estadio clínico). La segunda parte del instrumento consistió en la instrucción y una matriz en la que se presentaron el ítem y la escala de respuesta; por cada uno de los ítems se indagó por dificultad, confusión, palabras difíciles, percepción de sentirse molesto u ofendido por el contenido del ítem —en este caso 
se incluyó el parafraseo sugerido por el paciente, cuando este lo proporcionó-. Adicionalmente, hubo un espacio destinado a comentarios. En general, los ítems fueron comprendidos por los pacientes. Aquellos que ocasionaron mayor confusión fueron los ítems que incluyen negación: 2, 4, 6, 9, 13, 14 (ver Anexo). La recomendación más frecuente fue redactar el ítem de forma positiva pues, en español, las negaciones requieren de un mayor esfuerzo cognitivo para pensar la respuesta en escala Likert $\mathrm{y}$, adicionalmente, es una estructura gramatical poco frecuente. Sin embargo, la modificación de los ítems a un sentido positivo afecta el sentido del ítem original e implica una transformación de la escala de calificación, lo que daría lugar a una prueba diferente. Por lo tanto, se decidió mantener la estructura gramatical original. El ítem 14: "Preferiría no estar vivo", produjo reacciones de molestia-ofensa entre los participantes. Con frecuencia mencionaron que, si prefirieran no estar vivos, no estarían en la institución buscando cómo sentirse mejor y superar las consecuencias de la enfermedad. En la versión definitiva de la prueba, se ajustó el género en la redacción para cada palabra que así lo requiriera. No fue necesario realizar otros ajustes gramaticales a la versión traducida del instrumento (ver Anexo), por lo que se procedió a aplicarlo en la siguiente fase del estudio.

\section{Características de la muestra}

La muestra estuvo conformada por 516 pacientes oncológicos en cuidado paliativo, $313(60.66 \%) \mathrm{mu}-$ jeres y 203 (39.34\%) hombres, con una mediana de edad de 60 años (RIC= 17,5 años; rango de edad 21 a 86 años); casado (32.56\%), en unión libre (21.90\%) o soltero $(24.03 \%)$ y con una mediana de 2 hijos (RIC $=3$ hijos; rango entre 0 y 15 hijos). Los pacientes fueron procedentes de diferentes regiones del país, especialmente de zona urbana (82.95\%), provenientes de Bogotá — capital del país- (31.59\%), Cundinamarca (19.19\%), Boyacá (16.09\%) y Tolima-Huila (11.63\%). La mayoría de los pacientes reportaron vivir con su familia nuclear $(66.28 \%)$ o con su pareja (20.35\%), en viviendas ubicadas en estratos socioeconómicos bajo (70.93\%) y medio $(25.58 \%)$ y tener bajos niveles educativos $(77.72 \%)$. Incluso, $5.04 \%$ de los pacientes reportaron que nunca estudiaron. El $71.71 \%$ declararó estar laboralmente inactivo, el 14.15\% laboralmente activo y el $14.15 \%$ pensionado.

Con base en los criterios establecidos por Robinson et al. (2016a), se encontró que $45.35 \%$ de los participantes tenían niveles moderados de desmoralización, $28.29 \%$, altos y $26.36 \%$, bajos.

Para la opción de respuesta "nunca", los ítems con mayor puntaje fueron (p13) Soy una persona que no vale la pena (87\%); (p5) Siento que nadie puede ayudarme (79\%); (p6) Siento que no puedo ayudarme (78\%), y (p9) Siento que no afronto bien la vida (77\%). Para la opción de respuesta "Algunas veces", los ítems con mayor puntaje fueron (p11) Tiendo a sentirme fácilmente afectado(a) en el aspecto emocional (40\%); (p12) Me siento afligido(a)/acongojado(a) por lo que me está pasando (47\%), y (p8) Me siento irritable (48\%). Para la opción de respuesta "muchas veces", los ítems con mayor puntaje fueron (p10) Me arrepiento de muchas cosas en la vida (17\%); (p8) Me siento irritable (18\%); (p11) Tiendo a sentirme fácilmente afectado(a) en el aspecto emocional (20\%), y (p12) Me siento afligido(a)/ acongojado(a) por lo que me está pasando (24\%).

\section{Análisis factorial}

Análisis factorial exploratorio. Los resultados del test de esfericidad de Bartlett $\left(\chi^{2}(120)=\right.$ $2523.843, \mathrm{p}<0.0001)$ y del test $\mathrm{KMO}(\mathrm{KMO}=0.900)$ sugieren una adecuada estructura de la matriz de correlación. La estructura del gráfico de sedimentación de valores propios y test de análisis paralelo sugirió una solución óptima con 3 factores. La estructura con rotación ortogonal (varimax) fue la que ofreció una interpretación más consistente (tabla 1). Como puede observarse, el ítem 10 (p10 
"Me arrepiento de muchas cosas en la vida") no obtiene una adecuada carga factorial en ninguno de los tres dominios y muestra los valores de unicidad más altos. F1 representa falta de control emocional a través de seis ítems (p11, p12, p16, p4, p15, p8). F2 recoge características propias de sintomatología depresiva, representadas principalmente por ideación de desesperanza, minusvalía y muerte, a través de siete ítems (p13, p9, p14, p7, p1,p6,p5). F3 está representado solamente por dos ítems (p2 y p3) y se relaciona con aspectos del sentido de la vida.

Análisis factorial confirmatorio. La figura 1 representa el sistema de ecuaciones estructurales correspondiente al análisis factorial confirmatorio de la DS-II efectuado con 320 observaciones. Las formas circulares representan los dominios, las formas cuadradas los ítems, las flechas con una sola dirección las relaciones causales dominio-ítem y las flechas con doble dirección las correlaciones entre factores. Los estimadores del modelo de ecuaciones estructurales $\left(\chi^{2}=52.88 ; d f=87\right.$; RMSEA $=0.0001 ; \mathrm{NFI}=0.982 ; \mathrm{CFI}=0.999 ; \mathrm{SRMR}=$ 0.048 ) sugieren un adecuado ajuste del modelo con los tres dominios ( $\mathrm{Snt}=$ sentido de la vida; $\mathrm{Emc}=$ falta de control emocional; Dpr= sintomatología depresiva).

Tabla 1

Estructura factorial de la escala DS-II

\begin{tabular}{|c|c|c|c|c|c|}
\hline & Ítems & F1 & $\mathrm{F} 2$ & F3 & $\mathrm{u} 2 *$ \\
\hline p11 & Tiendo a sentirme fácilmente afectado(a) en el aspecto emocional & 0.72 & & & 0.46 \\
\hline p12 & Me siento afligido(a)/acongojado(a) por lo que me está pasando & 0.65 & & & 0.48 \\
\hline p16 & Me siento atrapado(a) por lo que me está pasando & 0.46 & & & 0.63 \\
\hline $\mathrm{p} 4$ & Siento que ya no controlo mis emociones & 0.44 & & & 0.66 \\
\hline p15 & Me siento muy solo(a) o aislado(a) & 0.40 & & & 0.71 \\
\hline p8 & Me siento irritable & 0.39 & & & 0.83 \\
\hline p10 & Me arrepiento de muchas cosas en la vida & 0.26 & & & 0.88 \\
\hline p13 & Soy una persona que no vale la pena & & 0.67 & & 0.42 \\
\hline p9 & Siento que no afronto bien la vida & & 0.61 & & 0.56 \\
\hline p14 & Preferiría no estar vivo(a) & & 0.51 & & 0.57 \\
\hline p7 & Me siento desesperanzado(a) & & 0.46 & & 0.54 \\
\hline p1 & Siento que tengo pocas cosas buenas para ofrecer a los demás & & 0.42 & & 0.69 \\
\hline p6 & Siento que no puedo ayudarme & & 0.40 & & 0.79 \\
\hline p5 & Siento que nadie puede ayudarme & & 0.35 & & 0.74 \\
\hline p3 & Siento que mi papel en la vida se ha perdido & & & 0.70 & 0.37 \\
\hline p2 & Parece que mi vida no tiene sentido & & & 0.67 & 0.37 \\
\hline
\end{tabular}

Nota. * U2: unicidad 


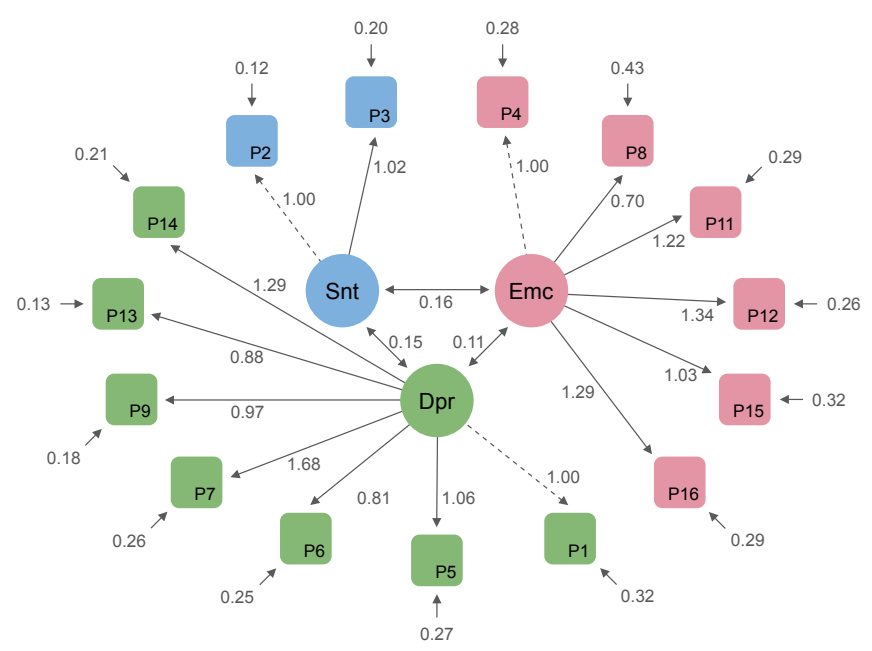

Figura 1. Sistema de ecuaciones estructurales, escala DS-II

Consistencia interna. El coeficiente alfa de Cronbach para la DS-II (versión español-Colombia) fue de 0.87 (IC95\%=0.86 a 0.89). Para F1 el alfa de Cronbach fue de 0.75 , mientras que para F2 y F3 fue 0.78. El valor del coeficiente lambda de Guttman para la DS-II total fue 0.88 . La tabla 2 muestra los valores de los dos coeficientes calculados con retiro de cada uno de los ítems. Como puede observarse, ninguno de los valores se incrementa al retirar alguno de los ítems.

Tabla 2.

Medidas de consistencia interna con retiro de cada uno de los items

\begin{tabular}{ccc}
\hline Ítem & Alfa de Cronbach & Lambda de Guttman \\
\hline $\mathrm{p} 1$ & 0.87 & 0.88 \\
\hline $\mathrm{p} 2$ & 0.86 & 0.87 \\
\hline $\mathrm{p} 3$ & 0.86 & 0.87 \\
\hline $\mathrm{p} 4$ & 0.86 & 0.88 \\
\hline $\mathrm{p} 5$ & 0.87 & 0.88 \\
\hline $\mathrm{p} 6$ & 0.87 & 0.88 \\
\hline $\mathrm{p} 7$ & 0.86 & 0.87 \\
\hline $\mathrm{p} 8$ & 0.87 & 0.88 \\
\hline $\mathrm{p} 9$ & 0.86 & 0.88 \\
\hline $\mathrm{p} 10$ & 0.87 & 0.88 \\
\hline $\mathrm{p} 11$ & 0.86 & 0.87 \\
\hline $\mathrm{p} 12$ & 0.86 & 0.87 \\
\hline $\mathrm{p} 13$ & 0.86 & 0.87 \\
\hline $\mathrm{p} 14$ & 0.86 & 0.87 \\
\hline $\mathrm{p} 15$ & 0.86 & 0.88 \\
\hline $\mathrm{p} 16$ & 0.86 & 0.87 \\
\hline
\end{tabular}


Validez de criterio concurrente. El coeficiente de correlación de Pearson entre los puntajes totales de las dos escalas fue 0.74 , significativamente diferente de cero. Los mejores valores de correlación se dan con F1 (falta de control emocional, $\mathrm{r}=0.7$ ) (tabla 3).

Confiabilidad test-retest. La mediana de tiempo entre las dos evaluaciones fue de 6 días (RIC $=2$ días). Si bien todos los valores de correlación fueron significativamente diferentes de cero, ninguno de los coeficientes fue mayor que 0.64. El menor valor $(0.51)$ correspondió a la correlación pre-post del F2 (sintomatología depresiva) (ver tabla 4).
Los valores del coeficiente de correlación-concordancia de Lin entre las dos mediciones repetidas fueron para el puntaje total 0.56 (IC95\%: 0.44 a 0.67), y para F1: 0.55 (IC95\%: 0.43 a 0.67 ), F2: 0.46 (IC95\%: 0.32 a 0.60$)$ y F3: 0.57 (IC95\%: 0.45 a 0.70$)$.

Según los límites de acuerdo de Bland y Altman para la DS-II total y para cada uno de los dominios, excepto para F3, a mayores puntajes tiende a presentarse menor grado de acuerdo entre las evaluaciones pre y post. En el caso del F3 el grado de acuerdo no parece relacionarse con la magnitud de la medición (figura 2).

Tabla 3.

Coeficientes de correlación entre BDI-II y DS-II (Puntaje total y de dominios)

\begin{tabular}{lccccc}
\hline & Total F1 & Total F2 & Total F3 & Total DS-II & Total BDI-II \\
\hline Total F1 & 1 & & & \\
\hline Total F2 & 0.59 & 1 & 1 & & \\
\hline Total F3 & 0.54 & 0.57 & 0.74 & 1 & 1 \\
\hline Total DS-II & 0.89 & 0.85 & 0.60 & 0.74 & 1 \\
\hline Total BDI-II & 0.70 & 0.51 & & \\
\hline
\end{tabular}

Tabla 4.

Coeficientes de correlación entre medidas pre y post, escala DS-II

\begin{tabular}{|c|c|c|c|c|c|c|c|c|}
\hline & Total pre & Total post & F1 pre & F2 pre & F3 pre & F1 post & F2 post & F3 post \\
\hline Total pre & 1 & & & & & & & \\
\hline Total post & 0.64 & 1.00 & & & & & & \\
\hline F1 pre & 0.93 & 0.62 & 1.00 & & & & & \\
\hline F2 pre & 0.84 & 0.48 & 0.64 & 1.00 & & & & \\
\hline F3 pre & 0.81 & 0.59 & 0.66 & 0.63 & 1.00 & & & \\
\hline F1 post & 0.58 & 0.91 & 0.62 & 0.36 & 0.53 & 1.00 & & \\
\hline F2 post & 0.46 & 0.72 & 0.37 & 0.51 & 0.37 & 0.45 & 1.00 & \\
\hline F3 post & 0.54 & 0.77 & 0.50 & 0.37 & 0.62 & 0.65 & 0.40 & 1.00 \\
\hline
\end{tabular}



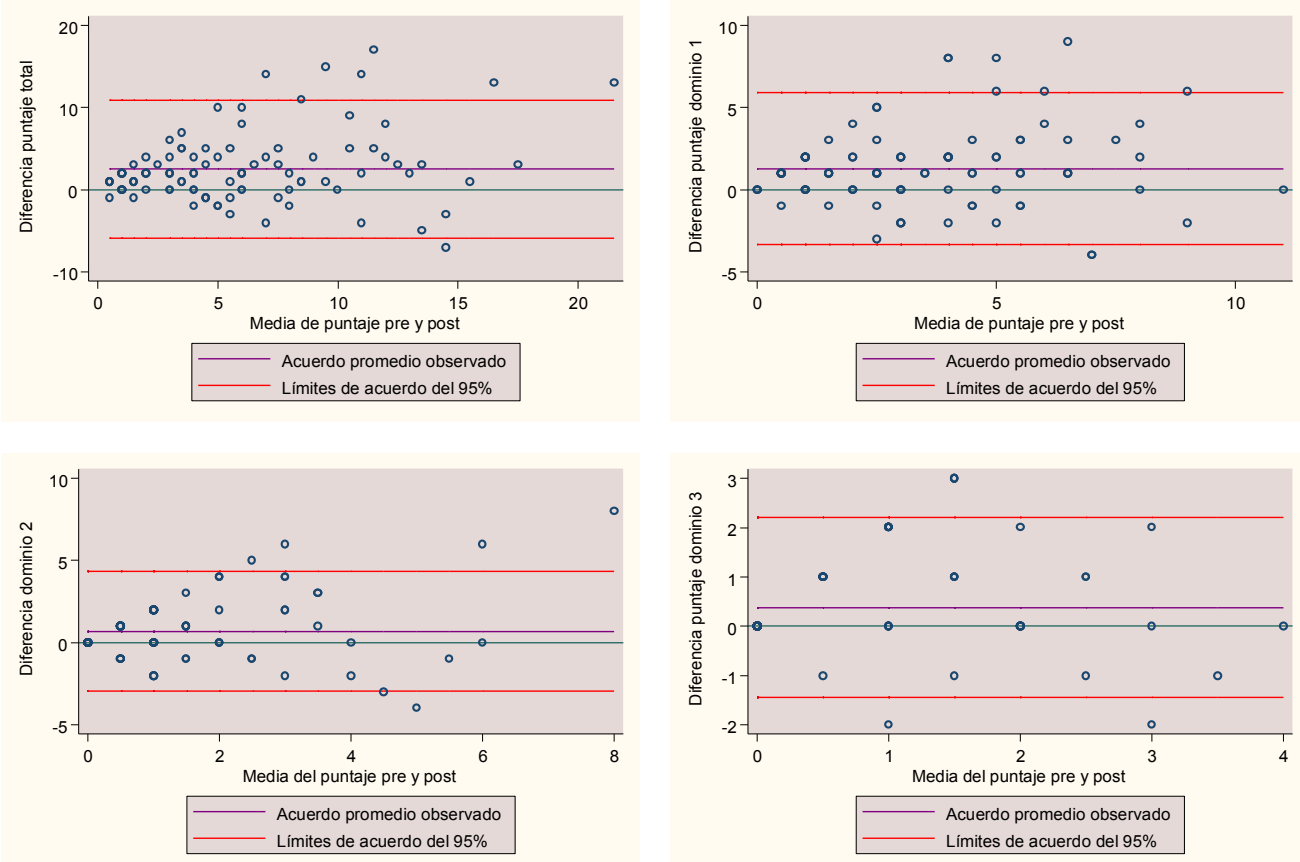

Figura 2. Límites de acuerdo de Bland y Altman

Análisis de Rasch. Los resultados del análisis de componentes principales de los residuos con porcentaje de varianza cruda explicada por la medición de $41.3 \%$ y eigenvalue de la varianza no explicada en el primer contraste de 1.86 sugieren la unidimensionalidad del instrumento. Esto se confirma con el hallazgo de valores de cuadrado medio de infit entre el rango de 0.6 a 1.5 y desviación estándar de coeficientes estandarizados menor que 2 (tabla 5).

En cuanto a la estimación de confiabilidad de personas e ítems, el coeficiente más bajo (correspondiente a las personas) es cercano a 0.8 . Al es- timar las medidas de separación, los valores para los ítems resultaron adecuados, mientras que, en el caso de las personas, los valores se pueden considerar bajos (tabla 5).

En lo referente a la calidad del ajuste de cada uno de los ítems al modelo, se encontraron valores sugestivos de mal ajuste en los ítems "Siento que no puedo ayudarme" y, especialmente, "Me arrepiento de muchas cosas en la vida" (tabla 6). Este último ítem había mostrado bajas cargas factoriales en el análisis de validez de constructo y el valor de unicidad más alto.

Tabla 5.

Valores de los cuadrados medios de infit y outfit y medidas de separación y confiabilidad de personas e items

\begin{tabular}{lcccccc}
\hline \multicolumn{2}{c}{ Infit } & \multicolumn{2}{c}{ Outfit } \\
\hline MNSQ* & ZSTD** & MNSQ* & ZSTD** & Separación & Confiabilidad \\
\hline Personas & 0.99 & 0.001 & 0.98 & 0.001 & 1.82 & 0.77 \\
\hline Ítems & 1.03 & 0.2 & 0.98 & -0.3 & 8.5 & 0.99 \\
\hline
\end{tabular}

Nota. * Cuadrado medio ** Desviación estándar de coeficientes estandarizados 
Tabla 6.

Medidas de ajuste de los items de la escala DS-II

\begin{tabular}{|c|c|c|c|c|c|c|}
\hline \multirow[b]{2}{*}{ Ítem } & \multirow[b]{2}{*}{ Puntaje } & \multirow[b]{2}{*}{ Medida* } & \multicolumn{2}{|c|}{ Infit } & \multicolumn{2}{|c|}{ Outfit } \\
\hline & & & MNSQ & ZSTD & MNSQ & ZSTD \\
\hline Siento que tengo pocas cosas buenas para ofrecer & 181 & 54.65 & 1.20 & 2.56 & 1.24 & 1.91 \\
\hline Parece que mi vida no tiene sentido & 222 & 51.42 & .90 & -1.48 & .72 & -2.94 \\
\hline Siento que mi papel en la vida se ha perdido & 281 & 47.39 & .91 & -1.39 & .79 & -2.49 \\
\hline Siento que ya no controlo mis emociones & 227 & 51.06 & 1.01 & .22 & .91 & -.86 \\
\hline Siento que nadie puede ayudarme & 145 & 57.95 & 1.25 & 2.76 & .92 & -.54 \\
\hline Siento que no puedo ayudarme & 144 & 58.05 & 1.24 & 2.72 & 1.57 & 3.47 \\
\hline Me siento desesperanzado(a) & 303 & 46.02 & .82 & -3.15 & .85 & -1.91 \\
\hline Me siento irritable & 435 & 38.58 & 1.14 & 2.49 & 1.30 & 3.99 \\
\hline Siento que no afronto bien la vida & 138 & 58.67 & .95 & -.57 & .84 & -1.13 \\
\hline Me arrepiento de muchas cosas en la vida & 342 & 43.70 & 1.39 & 5.95 & 1.64 & 6.98 \\
\hline Tiendo a sentirme fácilmente afectado(a) emocionalmente & 414 & 39.70 & .94 & -1.15 & .90 & -1.38 \\
\hline Me siento afligido(a)/ acongojado(a) & 490 & 35.68 & .72 & -5.73 & .72 & -4.59 \\
\hline Soy una persona que no vale la pena & 84 & 65.47 & .90 & -.89 & .54 & -2.68 \\
\hline Preferirla no estar vivo(a) & 170 & 55.60 & .97 & -.34 & .73 & -2.37 \\
\hline Me siento muy solo(a) o aislado(a) & 233 & 50.62 & 1.11 & 1.59 & 1.10 & .98 \\
\hline Me siento atrapado(a) por lo que me está pasando & 307 & 45.78 & .97 & -.55 & .91 & -1.09 \\
\hline
\end{tabular}

Nota. * La medida corresponde a logits transformados entre cero y 100.

En relación con la calidad de la medición de categorías de respuesta de los ítems, la medida en logits transformados varió entre -19.43 para la categoría 0, cero para la categoría 1 y 19.43 para la categoría dos. Como puede observarse en la figura 3, hay una adecuada ordenación en los puntajes y las probabilidades de las categorías.

Finalmente, el mapa de Wright (figura 4) permite observar que existe un grupo de pacientes con niveles del atributo mayores que los que puede medir el instrumento. La diferencia entre la media del puntaje entre pacientes e ítems (más de 30 logits transformados en escala de 0 a 100) indica que la muestra de pacientes presenta niveles del atributo con una intensidad más alta que la que mide el instrumento; adicionalmente, se observa que la intensidad del atributo en un grupo considerable de pacientes con niveles altos y bajos del atributo no alcanza a ser medida por la DS-II. 
CATEGORY PROBABILITIES: MODES - Andrich thresholds at intersections

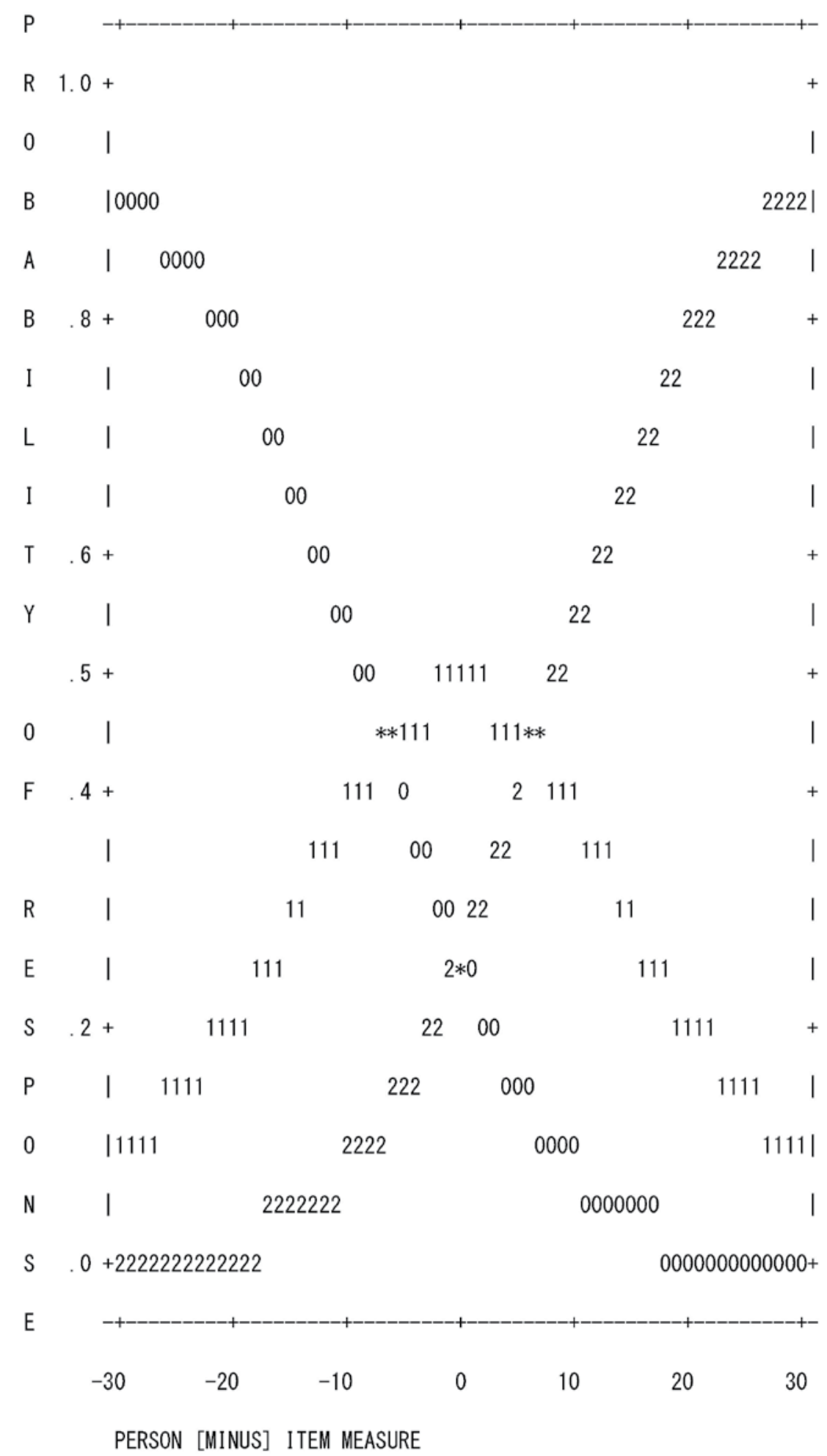

Figura 3. Calidad de la medición de las categorías de los ítems 


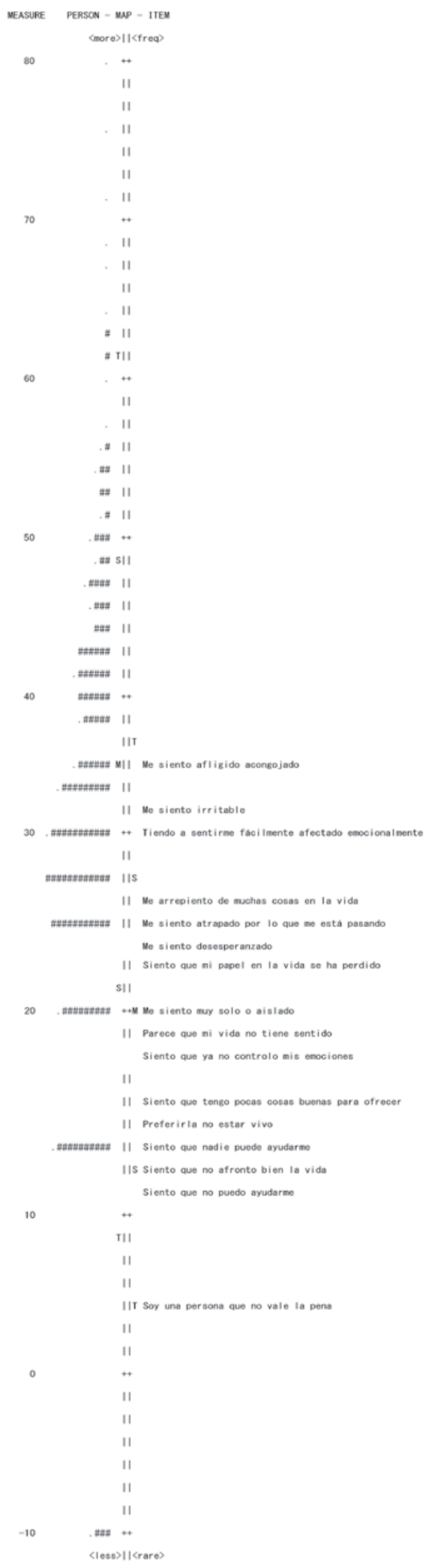

Figura 4. Mapa de Wright, escala DS-II 


\section{Discusión}

Si bien la desmoralización es un síndrome plenamente descrito y con una alta frecuencia entre pacientes oncológicos que reciben cuidado paliativo, en Colombia no disponemos de un instrumento para medirlo y explorarlo con rigor, en los pacientes con cáncer avanzado. El objetivo del presente estudio fue analizar las propiedades psicométricas de la DS-II (versión español-Colombia) en una muestra de 516 pacientes con cáncer en cuidado paliativo.

El análisis factorial exploratorio mostró la presencia de tres factores o dominios (Snt; Emc y Dpr), lo que difiere del instrumento original en el que solo se describen dos subescalas: distrés y habilidad de afrontamiento (11 ítems) y significado y propósito (11 ítems) (Robinson et al., 2016b).

Es pertinente considerar que la sintomatología depresiva es un componente de muchos trastornos, incluso de enfermedades orgánicas, como el cáncer. Lo resultados del presente estudio indican que la desmoralización tiene dentro de su estructura un componente dado por algunos síntomas depresivos, lo que está a favor de la propuesta de que depresión - como constructo clínico y no como síntomas- $-\mathrm{y}$ desmoralización son categorías diferentes.

Se encontró que el ítem 10 no obtuvo adecuada carga factorial en ninguno de los tres dominios, al retirarlo no se disminuyen los indicadores de consistencia interna del instrumento y muestra un mal ajuste dentro del modelo de Rasch. Es probable que la forma en que está escrito el ítem, "Me arrepiento de muchas cosas en la vida", dé lugar a una interpretación que genere reactancia en el paciente pues lleva implícito un mensaje de que se han hecho mal muchas cosas en la vida y, además, el arrepentimiento es un proceso afectivo-cognitivo que, en un país con alta tendencia religiosa cristiana, supone reflexión y compromiso espiritual. Puede considerarse que, en una situación de final de vida, este tipo de evaluaciones impliquen alto costo emocional que no todos los pacientes están dispuestos a asumir. Adicionalmente, consideramos que este es un ítem que, a diferencia de los demás que componen la escala, implica hacer una reflexión en retrospectiva, lo que también pudo tener algún efecto sobre la respuesta. Sería interesante considerar si, para el contexto colombiano, esta pregunta realmente evalúa el síndrome de desmoralización. Finalmente, como también lo indicó Karabatsos (2000), es pertinente recordar que la falta de ajuste del ítem puede también explicarse por sesgo del ítem, respuestas al azar, baja motivación o bajo interés de cooperar.

El análisis factorial confirmatorio, respalda la presencia de los tres dominios mencionados y muestra que el ajuste de la DS-II es aceptable.

Los análisis de consistencia interna muestran que la DS-II (versión español-Colombia) es confiable, al igual que cada uno de sus dominios de manera independiente, aunque el coeficiente alfa de Cronbach es menor que en la DS-II para los tres dominios hallados en este análisis. Los coeficientes alfa de Cronbach y lambda de Gutman son de 0.87 y 0.88 respectivamente, para la DS-II total.

Si bien esta y el BD-II correlacionaron, F2 (sintomalogía depresiva) mostró una correlación modesta (0.51), lo que indica que, a pesar de estar relacionadas, la depresión y la desmoralización son dos condiciones clínicas independientes.

En cuanto a la confiabilidad test-retest, se encontró que la correlación no es buena. Sin embargo, esto no significa que la DS-II (versión español-Colombia) no sea confiable ni inestable en la capacidad de medición de la desmoralización, sino que la condición clínica de los pacientes es altamente variable lo que puede afectar la estabilidad del constructo. Por lo tanto, si el paciente respondía la prueba inicialmente estando muy indispuesto, con dolor, sufrimiento, etc., y luego volvía a responder el retest con un franco alivio del cuadro descrito, la puntuación difería notoriamente. Por lo tanto, es fundamental tener presentes estas variables en la medición del síndrome de desmoralización. 
Futuros estudios pueden indagar si el instrumento se comporta de igual manera en pacientes colombianos con cáncer en estadios tempranos de la enfermedad, como lo evaluaron en su estudio Vehling y Mehnert (2014), en los que la proximidad con la muerte se percibe más lejana y por lo tanto, los pacientes se sienten con mayores expectativas de vida y esperanza. Es de anotar que, de acuerdo con Clarke y Kissane (2002), la desesperanza es un elemento característico de la desmoralización y está asociada con un mal pronóstico, como es el caso de los participantes en nuestro estudio.

De hecho, si lo anterior se confirmara, sería coherente con los resultados de Vehling y Mehnert (2014), según los cuales la desmoralización está estrechamente asociada con los síntomas físicos experimentados por el paciente oncológico — que suelen ser múltiples e intensos en el paciente paliativo-, pero estos a la vez están mediados por la percepción de pérdida de dignidad, también común en los pacientes que reciben cuidado paliativo.

Una limitación del estudio está en no haber podido analizar la validez de criterio concurrente con un instrumento que mida específicamente la desmoralización, pero en la actualidad no se dispone de otro instrumento que evalúe este síndrome.

A través del análisis de Rasch se evidenció que la DS-II (versión español-Colombia) es un instrumento que permite captar niveles intermedios de desmoralización. Esto quiere decir que el instrumento no es suficientemente sensible para estimar niveles muy altos ni niveles muy bajos de desmoralización en esta población de pacientes oncológicos colombianos. Por lo tanto, se requiere hacer ajustes al instrumento para aumentar su sensibilidad a todos los niveles de desmoralización. En conclusión, se considera que la DS-II (versión español-Colombia) es un instrumento útil para identificar el síndrome de desmoralización en grado moderado. Sin embargo, es posible que el ítem 10, debido a su mal ajuste, deba ser retirado del instrumento o refraseado para determinar su calidad. De acuerdo con lo anterior, la DS-II (versión
español-Colombia) podría ser utilizada en el marco de una evaluación psicooncológica exhaustiva del estado mental del paciente adulto con cáncer, que recibe cuidado paliativo. Es recomendable aplicar la escala a manera de entrevista, especialmente en poblaciones con bajo nivel educativo, teniendo en cuenta que la aplicación puede tomar entre 10 y 15 minutos.

Dadas las dificultades de la DS-II (versión español-Colombia) para identificar pacientes escasamente o altamente desmoralizados, la evaluación psicológica rigurosa, debe hacerse siempre ante la sospecha o presencia de desmoralización. Es fundamental y necesario diseñar nuevos ítems para lograr la identificación de la desmoralización en toda la intensidad, especialmente en su presentación más alta. En caso de utilizarse esta versión en español de Colombia de la DS-II, debe hacerse con la mayor precaución la interpretación de los resultados, siempre por parte de un profesional y con la claridad de las limitaciones previamente expuestas.

\section{Referencias}

Beck, A.T., Steer, R.A., \& Brown, G.K. (1996). Manual for the Beck Depression Inventory-II. San Antonio, TX: Psychological Corporation.

Carvajal, A., Centeno, C., Watson, R., Martínez, M., \& Sanz Rubiales, A. (2011). ¿Cómo validar un instrumento de medida de la salud? Anales del sistema sanitario de Navarra, 34(1), 6372. http://scielo.isciii.es/scielo.php?script=sci arttext\&pid=S1137-66272011000100007\&1ng=es\&tlng=es

Clarke, D. M., \& Kissane, D. W. (2002). Demoralization: its phenomenology and importance. Australian \& New Zealand Journal of Psychiatry, 36(6), 733-742. https://doi.org/10.1046/j.14401614.2002.01086.x

Costantini, A., Picardi, A., Brunetti, S., Trabucchi, G., Bersani, F. S., Minichino, A., \& 
Marchetti, P. (2013). La versione italiana della Demoralization Scale: uno studio di validazione. Rivista di psichiatria, 48(3), 234-239. http://www.rivistadipsichiatria.it/r. php? $\mathrm{v}=1292 \& \mathrm{a}=14291 \& \mathrm{l}=20533 \& \mathrm{f}=$ allegati/01292_2013_03/fulltext/7-Costantini $\% 20$ (234-239).pdf

Dewolf, L., Koller, M., Velikova, G., Johnson, C., Scott, N., \& Bottomley, A. (2009). Eortc Quality of Life Group Traslation Procedure. 3 ed. EORTC.

Dimitrov, D. M., \& Smith, R. M. (2006). Adjusted Rasch person-fit statistics. Journal of Applied measurement, 7(2), 170.

Fisher, G.H., \& Molenaar, I.W. (eds.). (1995). Rasch Models. Foundations, Recent Developments, and Applications. Springer-Verlag.

Fox, C.M., \& Bond, T. (2015). Applying the Rasch Model. Fundamental Measurement in the Human Sciences. Third Edition. Taylor and Francis.

Grass, L., \& de Figueiredo, J. M. (2018). Advances in the Understanding of Demoralization in Oncology and Palliative Care. Psycho-Oncologie, 12(2), 99-108. https://doi.org/10.3166/ pson-2018-0049

Hung, H. C., Chen, H. W., Chang, Y. F., Yang, Y. C., Liu, C. L., Hsieh, R. K., ... \& Liu, S. I. (2010). Evaluation of the reliability and validity of the Mandarin Version of Demoralization Scale for cancer patients. Journal of Internal Medicine of Taiwan, 21(6), 427-435.

Karabatsos, G. (2000). A critique of Rasch residual fit statistics. Journal of Applied Measurement, 1(2), 152-176.

Kissane, D. W., Clarke, D. M., \& Street, A. F. (2001). Demoralization syndrome - a relevant psychiatric diagnosis for palliative care. Journal of palliative care, 17(1), 12-21.
Kissane, D. W., Wein, S., Love, A., Lee, X. Q., Kee, P. L., \& Clarke, D. M. (2004). The Demoralization Scale: a report of its development and preliminary validation. Journal of palliative care, 20(4), 269-276.

Lawrence, I., \& Lin, K. (1989). A concordance correlation coefficient to evaluate reproducibility. Biometrics, 45(1), 255-268.

Linacre, J. M. (2006). Rasch analysis of rank-ordered data. Journal of Applied Measurement, 7(1), 129-139.

Mehnert, A., Vehling, S., Höcker, A., Lehmann, C., $\&$ Koch, U. (2011). Demoralization and depression in patients with advanced cancer: validation of the German version of the demoralization scale. Journal of pain and symptom management, 42(5), 768-776. https://doi.org/10.1016/j. jpainsymman.2011.02.013

Mullane, M., Dooley, B., Tiernan, E., \& Bates, U. (2009). Validation of the Demoralization Scale in an Irish advanced cancer sample. Palliative \& supportive care, 7(3), 323-330. https://doi. org/10.1017/S1478951509990253

Robinson, S., Kissane, D. W., Brooker, J., \& Burney, S. (2015). A systematic review of the demoralization syndrome in individuals with progressive disease and cancer: a decade of research. Journal of pain and symptom management, 49(3), 595-610. https://doi.org/10.1016/j.jpainsymman.2014.07.008

Robinson, S., Kissane, D. W., Brooker, J., Hempton, C., Michael, N., Fischer, J., ... \& Burney, S. (2016a). Refinement and revalidation of the demoralization scale: The DS-II - external validity. Cancer, 122(14), 2260-2267. https://doi. org/10.1002/cncr.30012

Robinson, S., Kissane, D. W., Brooker, J., Michael, N., Fischer, J., Franco, M., ... \& Burney, S. (2016b). Refinement and revalidation of the 
demoralization scale: The DS-II-internal validity. Cancer, 122(14), 2251-2259. https://doi. org/10.1002/cncr.30015

Rudilla, D., Galiana, L., Oliver, A., \& Barreto, P. (2016). Demoralization Scale in Spanish-speaking palliative care patients. Journal of pain and symptom management, 51(4), 769-775. https:// doi.org/10.1016/j.jpainsymman.2015.11.019

Sanz, J., Perdigón, A. L., \& Vázquez, C. (2003). Adaptación española del Inventario para la Depresión de Beck-1l (BDI-II): 2. Propiedades psicométricas en población general [The
Spanish adaptation of Beck's Depression Inventory-11 (BDI-II): 2. Psychometric properties in the general population]. Clínica y Salud, 14(3), 249-280.

Vehling, S., \& Mehnert, A. (2014). Symptom burden, loss of dignity, and demoralization in patients with cancer: a mediation model. Psycho-Oncology, 23(3), 283-290. https://doi.org/10.1002/ pon. 3417

Wackerly, D. D., Mendenhall III, W., \& Scheaffer, R. L. (2013). Estadística matemática con aplicaciones. Séptima edición. Cengage Learning. 


\section{Anexo}

\section{Escala de Desmoralización (DS-II) versión en español (Colombia) para pacientes oncológicos que reciben cuidado paliativo}

A continuación, encontrará una serie de afirmaciones. Por favor indique qué tanto se ha sentido así en las últimas dos semanas. Marque el número correspondiente.

\begin{tabular}{|c|c|c|c|}
\hline & $\begin{array}{c}\text { Nunca } \\
0\end{array}$ & $\begin{array}{c}\text { Algunas veces } \\
1\end{array}$ & $\begin{array}{c}\text { Muchas veces } \\
2\end{array}$ \\
\hline \multicolumn{4}{|c|}{ 1. Siento que tengo pocas cosas buenas para ofrecer a los demás } \\
\hline \multicolumn{4}{|l|}{ 2. Parece que mi vida no tiene sentido. } \\
\hline \multicolumn{4}{|l|}{ 3. Siento que mi papel en la vida se ha perdido. } \\
\hline \multicolumn{4}{|l|}{ 4. Siento que ya no controlo mis emociones. } \\
\hline \multicolumn{4}{|l|}{ 5. Siento que nadie puede ayudarme. } \\
\hline \multicolumn{4}{|l|}{ 6. Siento que no puedo ayudarme. } \\
\hline \multicolumn{4}{|l|}{ 7. Me siento desesperanzado(a). } \\
\hline \multicolumn{4}{|l|}{ 8. Me siento irritable. } \\
\hline \multicolumn{4}{|l|}{ 9. Siento que no afronto bien la vida. } \\
\hline \multicolumn{4}{|l|}{ 10. Me arrepiento de muchas cosas en la vida. } \\
\hline \multicolumn{4}{|c|}{ 11. Tiendo a sentirme fácilmente afectado(a) en el aspecto emocional } \\
\hline \multicolumn{4}{|l|}{ 12. Me siento afligido/acongojado por lo que me está pasando. } \\
\hline \multicolumn{4}{|l|}{ 13. Soy una persona que no vale la pena. } \\
\hline \multicolumn{4}{|l|}{ 14. Preferiría no estar vivo(a). } \\
\hline \multicolumn{4}{|l|}{ 15. Me siento muy solo(a) o aislado(a). } \\
\hline 16. Me siento atrapado(a) por lo que me está pasando. & & & \\
\hline
\end{tabular}

